\section{The solar wind interaction}

\section{from P.A. Cloutier and C.T. Russell}

VENUS provides our only well documented example of the interaction of supersonic or, more correctly, supermagnetosonic magnetized plasma with an unmagnetized and highly-electrically conducting ionosphere. The Pioneer Venus observations confirm the zero-order picture derived from the earlier Venera and Mariner missions as sketched in Fig.1. A bow shock forms in front of the planet which heats, slows and deflects the flow around the ionosphere. The magnetic field is draped over the obstacle and increases in strength towards the planet while at the same time slipping around the planet as the plasma flows out of the plane of the figure. The physics by which this behaviour takes place is fairly well understood. The interesting physics, and that unique to Venus, at least so far, involves perturbations of this picture. These perturbations provided much of the controversy in the discussions of the solar wind interaction on the last day of the Venus conference.

Venus has a magnetic tail very like a comet. The Pioneer Venus magnetometer shows that behind Venus, in a region about 4 Venus radii across, the magnetic field is parallel to the solar wind flow and enhanced above interplanetary levels. Barnes (NASA Ames Research Center) and Intriligator (Carmel Research Center) reported that in the distant tail, interesting plasma effects are seen, including regions of depletion of solar wind plasma and regions of pick-up of ionospheric oxygen ions. Closer to the planet, Barnes observed the acceleration region for the oxygen pickup. Near the terminator, the oxygen flow was weakest and fastest at high altitudes, growing in density and decreasing in speed as the planet was approached.

The source of this oxygen is presumably charge exchange and photoionization of the hot neutral oxygen exosphere at altitudes above the normal ionosphere. At high altitudes, this new cold plasma is accelerated either by the solar wind electric field or by magnetic stresses and escapes from the planet. At low altitudes, in the ionosphere, the magnetic and electric fields are weak and new ions remain bound by the planet's gravitational field. It is difficult to assess how important these processes are. Vaisberg and Zeleny (Space Research Institute, Moscow) have modelled mass loading of field lines due to photoionization in a cylindrical geometry but find that the flux of oxygen is too low. Perhaps charge exchange will provide the

P.A. Cloutier is in the Department of Space Physics and Astronomy, Rice University, Houston, Texas 77001 , and C.T. Russell is in the Institute of Geophysics and Planetary Physics, University of California, Los Angeles, California 90024.

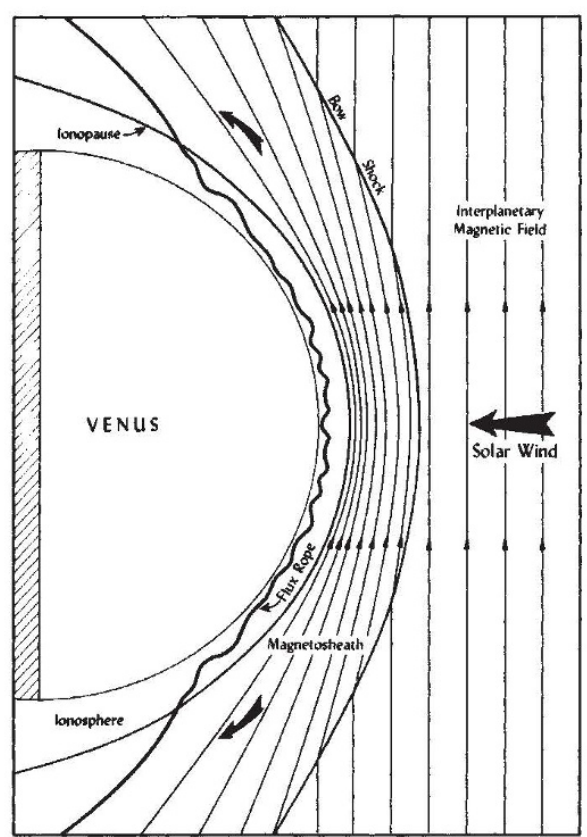

Fig. 1 Sketch of the interaction of the magnetized solar wind plasma with Venus's unmagnetized and highly-electrically conducting ionosphere.

missing flux but they prefer a source of anomalous ionization. Another indicator of the importance of these non-magnetohydrodynamic processes is the position (Slavin, UCLA, and Mihalov, NASA Ames Research Center) of bowshock. The shock is somewhat weaker and closer to Venus than would be expected if all the solar wind were deflected by the planet. Charge exchange could provide the requisite removal of momentum from the flow.

Fig. 2 Magnetic field line configuration on Venus's dayside (see the text). The dashed line shows the boundary separating regions stable and unstable to the Kelvin-Helmholtz instability.

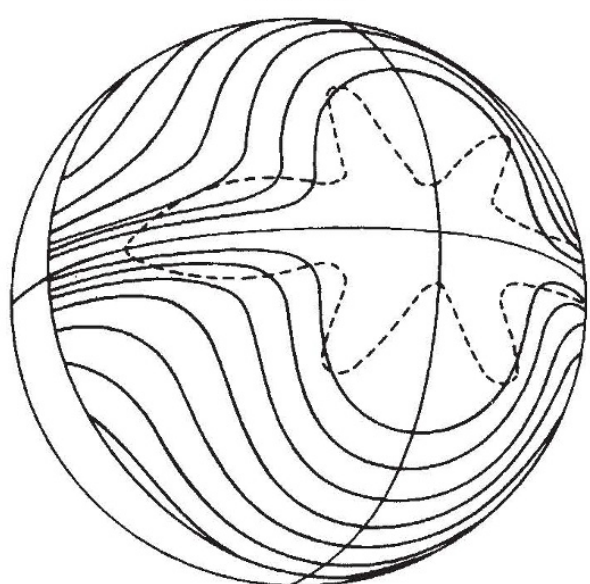

Some of these phenomena were anticipated by a minority of scientists before the latest barrage of data from Pioneer Venus. Many, however, were not. Two unexpected phenomena were discussed by Brace (NASA Goddard Space Flight Center): plasma clouds, dense regions of cold plasma apparently detached from the ionosphere proper in the terminator region and ionospheric holes, regions of density depletion accompanied by radial magnetic field in the near midnight ionosphere. Another unexpected feature of the dayside ionosphere is the existence of magnetic flux ropes, twisted bundles of magnetic flux which often pervade the dayside ionosphere. An artist's conception of the occurrence of such a rope is given in Fig.1.

Flux ropes and other observed features of the dayside ionospheric configuration may be explained as resulting from induced convection within the ionosphere driven by the solar wind interaction. In a model proposed by Cloutier (Rice University) and his colleagues, pressure gradients and electric fields induced in the ionosphere drive an anti-sunward convection pattern which carries ionization and the draped interplanetary magnetic field towards the anti-solar point. In the model, velocity shear within the ionospheric convection pattern leads to flux rope formation due to the onset of the Kelvin-Helmholtz instability. Calculations of the ion convection patterns, the field geometry and the extent of the Kelvin-Helmholtz unstable regions reproduce many of the observed characteristics of the dayside Venus magnetic field behaviour, including the presence or absence of flux ropes, the occurrence of large magnetic fields at low altitudes, and the lack of coincidence of times between the ionopause and large magnetic gradients. The field line configuration is shown in Fig. 2 by the solid lines, and the dashed contour shows the boundary between region stable and unstable to the Kelvin-Helmholtz instability. Flux rope formation may occur outside of the dashed contour.

The importance of the Kelvin-Helmholtz waves at the ionopause was discussed by Curtis (NASA Goddard Space Flight Center) who described the acceleration of ionosheath plasma by parallel electric fields arising from conversion of KelvinHelmholtz wave energy into a kinetic Alfvén wave. Curtis also showed that ions and electrons may be thermalized to high energies by high levels of plasma wave turbulence at Venus.

Finally, there are still some classical interaction problems to be solved: how, for example, does the flow close behind Venus (Knudsen, Lockheed), and is the interaction viscous rather than inviscid as preferred by Knudsen (Pérez-de-Tejada, Institute Geofísica, Mexico). In all there was a great deal of controversy and evidence of many more important principles to be uncovered in the solar wind-Venus interaction problem. 